\title{
Influence of fissure inclination and confining pressure on the local behaviour of natural clays
} \author{
Alessandra Castellano ${ }^{2}$, Francesco Massaro ${ }^{2}$ \\ ${ }^{1}$ Division of Geotechnical Engineering - LTH, Lund University, Lund, Sweden \\ ${ }^{2}$ DICATECh, Politecnico di Bari, Bari, Italy \\ ${ }^{3}$ Univ. Grenoble Alpes, CNRS, Grenoble INP, 3SR, F-38000 Grenoble, France \\ ${ }^{4}$ Division of Solid Mechanics - LTH, Lund University, Lund, Sweden
}

Erika Tudisco ${ }^{1, *}$, Claudia Vitone $^{2, * *}$, Cristina Mondello ${ }^{2}$, Gioacchino Viggiani ${ }^{3}$, Stephen A. Hall ${ }^{4}$, Federica $_{\text {Cotecchia }}{ }^{2}$,

\begin{abstract}
In this experimental study the influence of fissuring orientation and confinement pressure on the mechanical behaviour of natural clays is investigated. The tested material, the scaly clay from Santa Croce di Magliano (south of Italy), is characterised by an intense network of pre-existing fissures of single orientation. Several plane strain compression tests have been conducted, under different confinement pressures (i.e., from 50 to $600 \mathrm{kPa}$ ), on specimens having fissures with vertical, medium and horizontal inclination. Digital Image Correlation has been used to follow the deformation processes of the specimens throughout the tests by measuring incremental shear and volumetric strain maps. The results showed a strong coupling between the total confinement and the fissure inclination, that is controlling both the onset and the development of the patterns of the localisation processes. The new results have been compared with previous ones carried out on the same material without confinement. The comparison shed light on the role of total confinement that becomes particularly relevant from certain levels of pressures and fissuring inclination.
\end{abstract}

\section{Introduction}

Soils outcropping within chain areas have generally experienced long geological histories as a result of tectonism. Consequently, they often possess a 'complex' structure, which makes them behave rather differently from other natural soils. Such disturbed soils (usually referred to as structurally complex formations, [e.g. 1, 2]) are generally characterized by structural features such as fissuring and heterogeneities. Fissuring, in particular, makes a clay significantly weaker than an unfissured one [3] and may be responsible for severe engineering problems as, for example, slope instability $[4,1,5]$, or bulging of tunnel lining $[6,7]$.

The object of this study is a very highly fissured clay that outcrops in a small town within the southern Italian Apennines. It is an allochthonous clay, since it was originally deposited hundreds kilometres away from its current location and was subsequently subjected to large displacements during the Apennine orogenesis. It follows that its pre-existing fissures can be seen as the current product of the in-situ strain localisations phenomena induced by the large deformations experienced by the clay during its complex and long geological history. In particular, the fissures are organized into a peculiar intense network that splits the clay into millimetre lens-shaped elements (known as scales - which is the origin of their being referred to as scaly clays in the literature).

This paper reports a further step of a long lasting experimental research, which entails the experimental investigation of the geomechanical properties of several differently fissured clays, aiming at their modelling as a single class of geomaterials [8-13].

During previous laboratory studies, the SCM scaly clay [911] were tested in plane strain compression tests at zero confining pressure in the plane of deformation. The experiments have shown that, when the fissure orientation in the specimen is either random or horizontal, i.e., normal to the direction of the maximum principal stress, the development of localized deformation throughout plane strain compression tests, is not dictated by pre-existing fissures. When this is the case, for scaly clay specimens the shear band has been found to develop in a similar way to what observed for unfissured (intact) clays. Moreover, the tests have shown that, if fissure orientation is either $45^{\circ}$ or vertical, i.e., parallel to the direction of loading, the preexisting fissuring network takes control over the shearing behaviour of the material since early stages of deformation. Here-forth, the influence of both fissuring inclination and confinement pressure on global behaviour and strain localisation phenomena has been investigated. This has been pursued through the analysis of both the global stressstrain response during plane strain compression tests and the evolution of displacement and strain fields throughout the same tests, the latter carried out by means of 2D Digital Image Correlation (DIC). Plane strain (biaxial) tests with different confinement pressures have been conducted in Lund University (Sweden) on several SCM scaly clay specimens with different fissure inclinations. These new results could shed light into the coupling between the effect of confining pressure and fissure orientation on both the global response and the local patterns of soil behaviour.

\footnotetext{
*,** Corresponding author: erika.tudisco@,construction.lth.se, claudia.vitone@poliba.it
} 


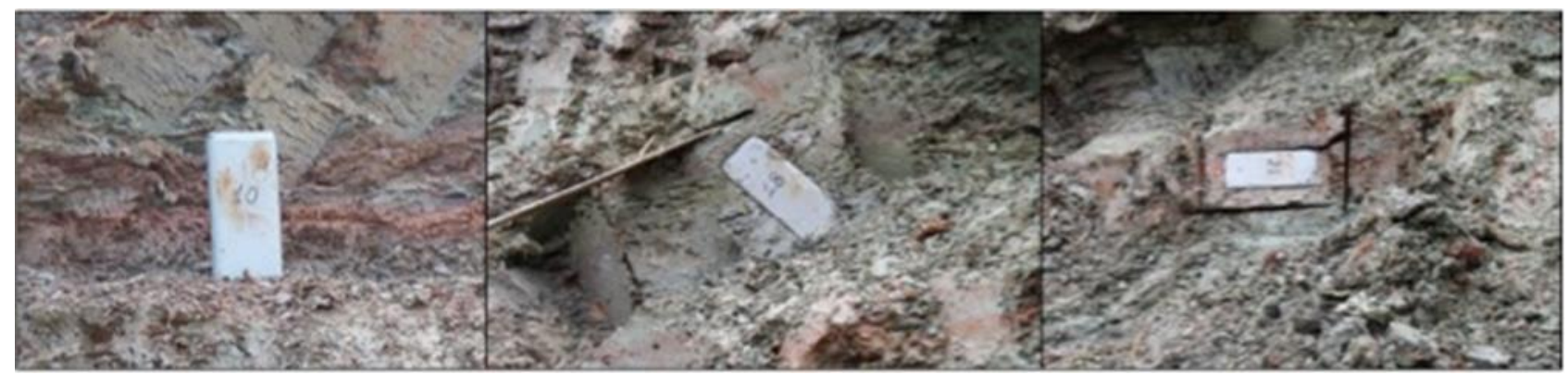

Fig. 1. Sampling by means of the C-shaped steel samplers. They were inserted in-situ with three different orientations.

\section{Material and sampling}

The tested fissured clay was block-sampled from an outcropping in a wide landslide basin in Santa Croce di Magliano (SCM hereafter), which is a small town of the Daunia region located within the north-eastern margin of the southern Italian Apennine. The scaly clay belongs to an Oligocene-Miocene Flysch succession of red and green scaly clays and calcareous strata (referred to as Red Flysch Formation by Dazzaro and Rapisardi [14], and Undifferentiated Clayey Complex by Melidoro et al. [15]).

As aforementioned, the SCM scaly clay is characterised by an intense fissuring network due to the extremely high shearing deformation experienced during its tectonic history. In particular, the fissures define a scaly meso-fabric, consisting of lens-shaped fragments of firm clay (the scales) of millimetre to centimetre size in length and millimetre size in thickness [8]. Each scale is in turn characterised by scale-in-scale fabric. The scales are not bonded between each other, but there is a strong bonding inside them (intra-scale bonding).

The samples were extracted within the in situ outcropping at about 3-4 meters below ground level, after removal of a thick stratum of soil. Due to the dimensional constraints of the biaxial apparatus (i.e., prismatic specimens $20 \mathrm{~mm}$ thick, width and height, in the plane of deformation, equal to 30 and $60 \mathrm{~mm}$, respectively) Cshaped steel samplers were on-purposely designed. This allowed us to both minimise the sampling disturbance and to make the fissures clearly visible on the specimen surface to be photographed throughout the plane strain tests [1617].

The samplers were inserted directly into the soil (Fig. 1) according to three different orientations in order to test, in the laboratory, specimens characterised by horizontal, $45^{\circ}$-inclined and vertical fissures. Moreover, they were inserted in-situ soon after lubricating their inner surface with silicon oil to minimise friction. Each sampler was then wrapped in cling film and waxed with paraffin in-situ, in order to minimise the water content loss.

The composition and physical properties of the scaly clay were determined in the geotechnical laboratory at the Politecnico di Bari (Italy), by following standard ASTM procedures (Table 1). The Table shows that the SCM clay is a silty clay of high plasticity and it can be classified as $\mathrm{CH}$ according to USCS soil classification. The high activity index (i.e., $\mathrm{A}=0.84$ ) appears to be consistent with the soil mineralogy that is mainly represented, within the clay fraction, by mixed layers of Illite/Smectite, where the Smectite content is predominant over that of Illite. The fissured mesofabric of the clay has been characterised within the characterization chart proposed by Vitone \& Cotecchia [8]. Specifically, the 'Fissuring IDentity chart' (F-ID chart, hereafter) of the SCM scaly clay shows that the scales are firm to soft (B3 to B4 in the chart), whereas the discontinuities are smooth to slickenside (D4 to D6), fresh to weathered (E1 to E3) and the fissure surfaces appear planar (G1) and characterized by many intersections $(\mathrm{H} 2)$. At the scale of a laboratory specimen, there is one single prevailing direction of the scales, so that also fissuring orientation is about single $\left(\mathrm{F} 1-\mathrm{X}^{\circ}\right.$, where $\mathrm{X}^{\circ}$ is the value, in degrees, of the fissure orientation to the horizontal). The fissuring intensity is the highest possible (I6) in the F-ID chart, due to both the small average volume of the scales and their very high specific surface (about 0.3 $\mathrm{cm}^{3}$ and $1000 \mathrm{~m}^{2} / \mathrm{m}^{3}$, respectively).

\section{Experimental setup and data interpretation}

The experiments with confinement pressure were performed at the laboratory of the department of Construction Sciences at Lund University, using confinement pressures of 50, 300 and $600 \mathrm{kPa}$, without any re-saturation prior to loading $[16,17]$. The results have been compared to previous plane strain tests, on the same material, without confinement pressure [11].

Previous to setting up, the samples were cut to the desired height, the free surface was rectified and smoothened, and the specimens were carefully extruded from the samplers (Fig. 2a-d).

In order to carry out the biaxial tests, a plane strain device was located in a MTS loading frame [18] (Fig. 2e). Two glass walls confined the front and back surfaces of the sample, and they guaranteed both the plane strain condition $\left(\varepsilon_{2}=0\right)$ and the visibility of the faces. The tests consisted first of an isotropic load and then of a shear load. The confinement pressure was applied by controlling the water pressure inside lateral membranes through a pressure regulator, whereas the vertical stress was controlled by the MTS machine. After the isotropic load, the shear load began by means of displacements control: the confinement pressure was maintained constant and the piston moved with a velocity of $0.006 \mathrm{~mm} / \mathrm{min}$. During the test, one of 
Table 1. Soil classification and mineralogical composition of the clay fraction. Key: Clay fraction (CF), Sand fraction (SF), silt fraction (MF), specific gravity of the soil (Gs), liquid limit $\left(\mathrm{w}_{\mathrm{L}}\right)$, plasticity index $(\mathrm{PI})$, average unit weight of soil $\left(\gamma_{\mathrm{av}}\right)$, average water content (wo), mixed layers illite/smectite (I/S), illite (I), kaolinite (K), chlorite (Ch). The illite content (I) is specified in brackets. *

Values from Vitone and Cotechia [8].

\begin{tabular}{|c|c|c|c|c|c|c|c|c|c|c|c|}
\hline $\mathrm{CF}$ & $\mathrm{SF}$ & $\mathrm{MF}$ & $\mathrm{G}_{\mathrm{s}}$ & $\mathrm{w}_{\mathrm{L}}$ & $\mathrm{PI}$ & $\gamma_{\mathrm{av}}$ & $\mathrm{w}_{0}$ & $\mathrm{I} / \mathrm{S} *$ & $\mathrm{I} *$ & $\mathrm{~K}^{*}$ & $\mathrm{Ch}^{*}$ \\
\hline$[\%]$ & {$[\%]$} & {$[\%]$} & {$[-]$} & {$[\%]$} & {$[\%]$} & {$\left[\mathrm{kN} / \mathrm{m}^{3}\right]$} & {$[\%]$} & {$[\%]$} & {$[\%]$} & {$[\%]$} & {$[\%]$} \\
\hline 74.00 & 2.00 & 24.00 & 2.77 & 99.00 & 61.93 & 17.94 & 28.54 & $80(30)$ & 1 & 17 & 2 \\
\hline
\end{tabular}
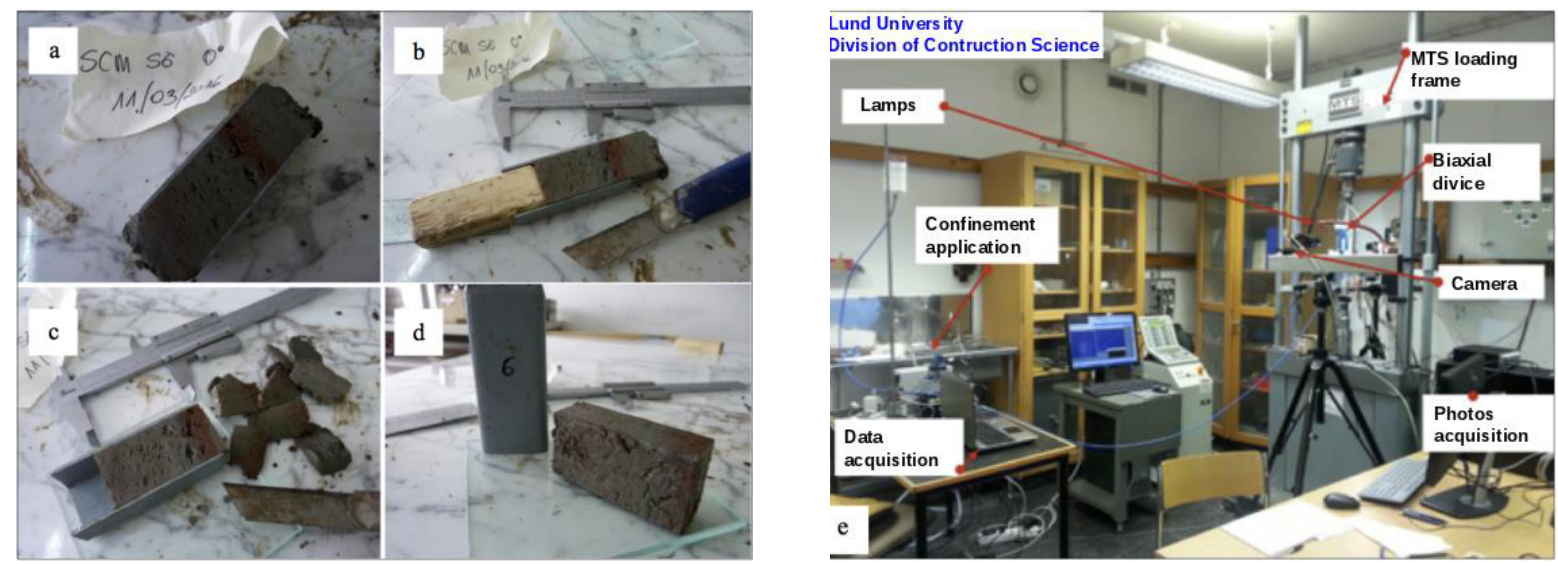

Fig. 2. Extraction of the sample from the mould: a) refinement of sample surface; b) c) refinement of its size and extraction; d) specimen ready for the test. e) Plane strain test setup: biaxial device, confinement applicator water pump, high resolution digital camera, cold lamps, computer for data and photo acquisition.

the visible face of the sample was photographed through an high resolution camera (29 Mpixel). Photos were acquired every 30 seconds during the confinement phase and every 20 minutes during the shearing phase.

The photographs have been analysed using 2D Digital Image Correlation (DIC) in order to follow the deformation processes throughout each test. DIC allows the stress-strain state to be related with the local deformation, within the specimen, by means of incremental shear and volumetric strain fields. In order to obtain a high quality DIC, it was necessary to realize speckles on the photographed face of the sample, using white and black coloured spray. The photographed surface of the specimens was illuminated using cold lamps in order to avoid overheating that would accelerate the reduction in saturation of the soil. In this case, the software TomoWarp 2 was used for the 2D-DIC (see [19-20]). This is a python-based code developed in cooperation between the University of Lund and the Laboratoire 3SR in Grenoble.

\section{Results}

Sixteen plane strain compression tests were carried out on the scaly clay specimens of different fissure inclinations. The tests were performed under three levels of confining pressures: 50, 300 and $600 \mathrm{kPa}$ without any re-saturation or consolidation of the specimen prior to loading (Table 2). The stress-strain curves of the new tests are compared in Fig. 3 with the biaxial test results carried out in Grenoble on the same clay at zero confining pressure (i.e., the minor principal total stress $\sigma_{3}$ was zero throughout each test; [8]). The results show that: i) the effect of confinement in general increases both the clay strength and stiffness; ii) for the same confining pressure the minimum strength is exhibited by specimens characterised by $45^{\circ}$-inclined fissures; iii) only specimens with vertical fissures exhibit a clear increase of the clay maximum strength as far as the confining pressure increases; iv) the global behaviour of specimens with horizontal fissures do not seem to be particularly sensitive to the level of confinement. Note that, in some cases (i.e., tests O4-300, O1-50 and I3-50), DIC revealed the presence of pre-existing 'second level fissures', i.e., more continuous and extended, not visible to the naked eye (Table 2). They were found to cross entirely the specimen, making the specimen size much smaller than the REV size, and to strongly influence both the development of strain localization end the global soil behavior. Moreover, specimen I2-50 was not homogeneous, since it included a stratum of marly clay.

Table 2. Testing programme. * data from Vitone et al. [11]

\begin{tabular}{|c|c|c|c|}
\hline \multirow[t]{2}{*}{ NAME OF THE TEST } & \begin{tabular}{|c|} 
FISSURE \\
INCLINATION TO \\
THE HORIZONTAL \\
DIRECTION \\
\end{tabular} & $\begin{array}{l}\text { CONFINING } \\
\text { PRESSURE - } \sigma_{3}\end{array}$ & \multirow[t]{2}{*}{ NOTES } \\
\hline & \begin{tabular}{|c|}
$\left.{ }^{\circ}\right]$ \\
\end{tabular} & {$[\mathrm{kPa}]$} & \\
\hline $\begin{array}{l}\text { V1-0* } \\
\end{array}$ & 90 & $\begin{array}{c}0 \\
\end{array}$ & \\
\hline V2-50 & 90 & 50 & \\
\hline V3-50 & 90 & 50 & \\
\hline $\begin{array}{l}\mathrm{V} 4-300 \\
\end{array}$ & 90 & 300 & \\
\hline V5-600 & 90 & 600 & \\
\hline V6-600 & 90 & 600 & \\
\hline$\left[11-0^{*}\right.$ & 45 & 0 & \\
\hline $12-50$ & 45 & 50 & marly strata \\
\hline $13-50$ & 45 & 50 & $2^{n d}$ level fissures \\
\hline $14-300$ & 45 & 300 & \\
\hline $15-600$ & 45 & 600 & \\
\hline IO- $0^{*}$ & $0-20$ & 0 & \\
\hline $01-50$ & 0 & 50 & $2^{\text {nd }}$ level fissures \\
\hline O2-50 & 0 & 50 & \\
\hline O3-50 & 0 & 50 & \\
\hline $04-300$ & 0 & 300 & $2^{\text {nd level fissures }}$ \\
\hline $05-300$ & 0 & 300 & \\
\hline O6-600 & 0 & 600 & \\
\hline $07-600$ & 0 & 600 & \\
\hline
\end{tabular}




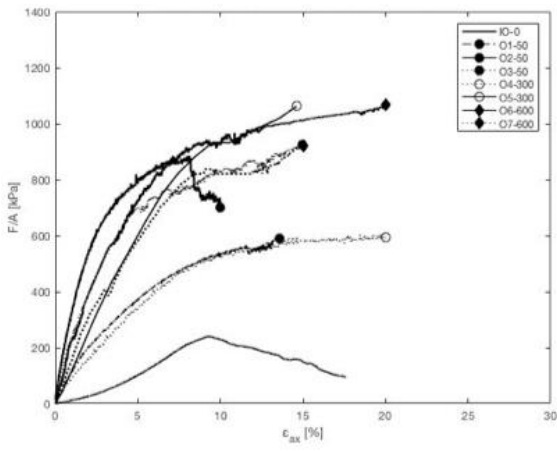

(a)

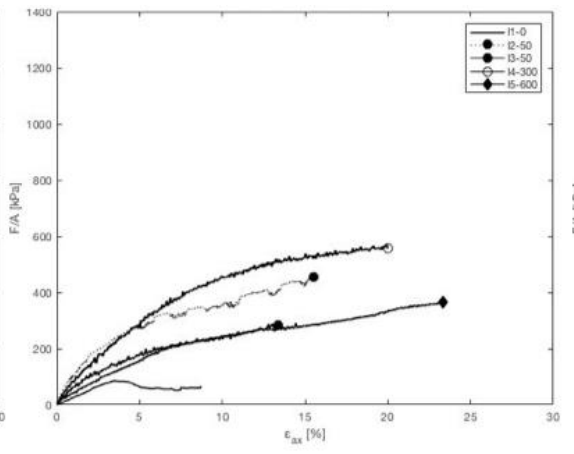

(b)

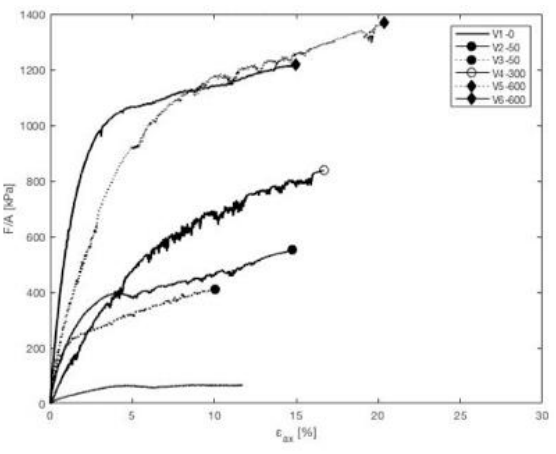

(c)

Fig. 3. Biaxial tests results on specimens having horizontal and sub-horizontal (a), medium (b) and vertical (c) fissuring inclination. Legend: $\bullet=50 \mathrm{kPa}, \mathrm{o}=300 \mathrm{kPa},=600 \mathrm{kPa}$ of confining pressure.

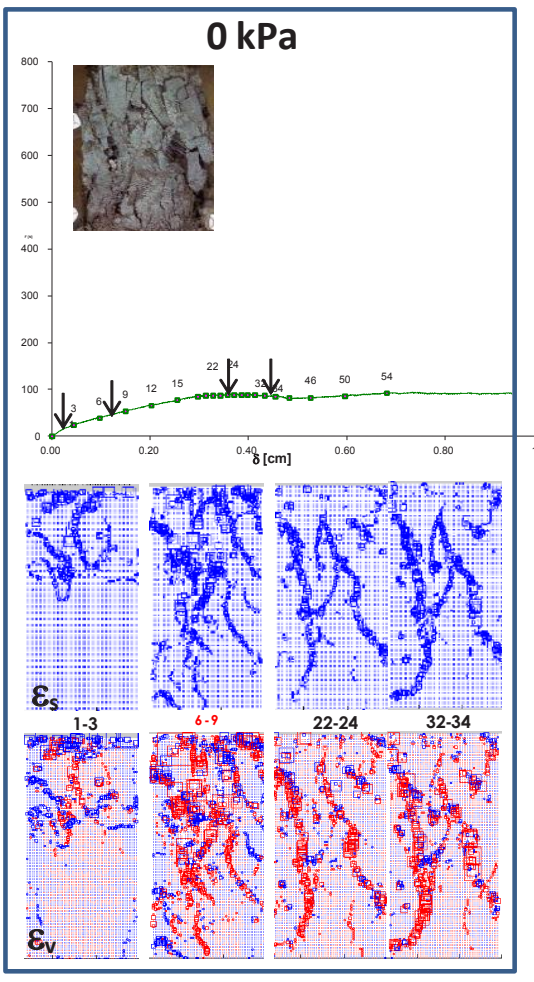

(a)

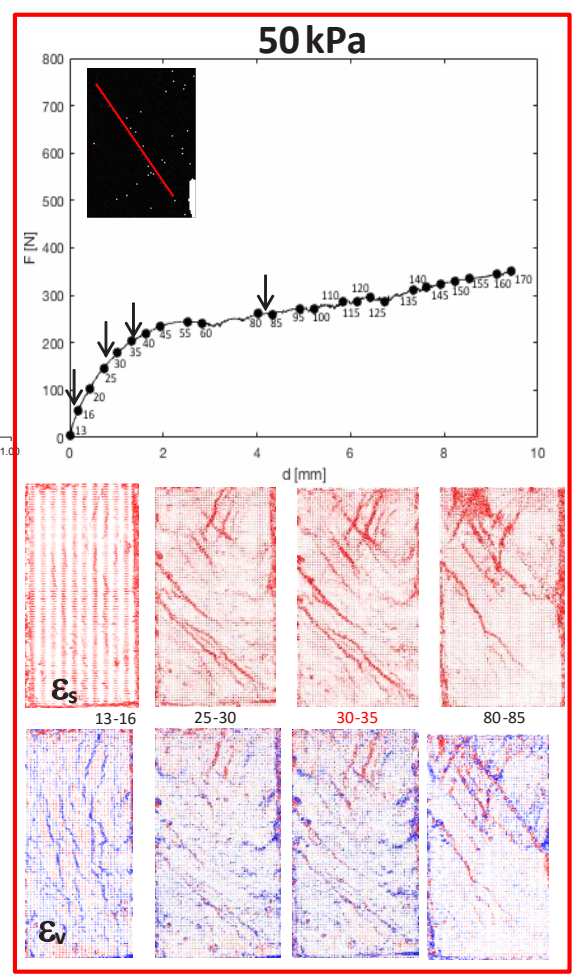

(b)

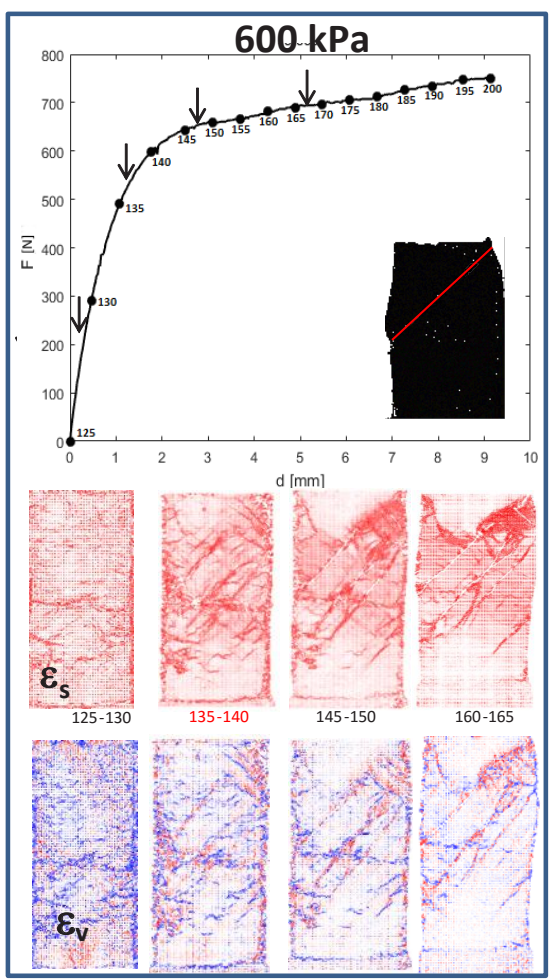

(c)

Fig. 4. V1-0 (a), V3-50 (b) and V6-600 (c) plane strain test results: load-displacement curves, DIC-derived shear and volumetric strain fields for selected load increments (arrows along the force-displacement curves) and specimens at the end of the tests.

Fig. 4a-c shows, for three scaly clay specimens having vertical fissures, the load-displacement curves, the DICderived shear and volumetric strain fields for selected load increments (arrows along the force-displacement curves), and the photographs of the specimens at the end of tests. Specifically, the coloured maps report the magnitude of the shear strains, $\varepsilon_{\mathrm{s}}$ (upper row), and of the volumetric strains, $\varepsilon_{\mathrm{v}}$ (bottom row; red symbols refer to dilation, the blue ones to contraction). The patterns of strain localisation of the tests with confinement significantly differ from that of test V1-0 (without confinement) where multiple pre-existing sub-vertical fissures are activated at the start of loading and the final failure mode is essentially axial splitting. In particular, it appears that the axial splitting failure mechanism along pre-existing fissure prevails in the test without confining pressure reducing the global strength (see V1-0, Fig. 4a). Both tests (Fig. 4. b-c) with confinement, instead, show that, at the beginning, the deformation is diffuse although several localized areas of fissure closure can be observed. This is particularly evident for test V3-50 where the closure of vertical fissures (in blue) is visible at increment 13-16. Before the change in curvature (about the maximum stress), deformation starts localizing or it becomes more intense in some areas. Localized deformation develops further as the soil deforms, leading eventually to a relative sliding mechanism mainly along a single sliding surface inclined about $55-60^{\circ}$ and $45-50^{\circ}$ to the horizontal for $\sigma_{3}$ equal to 50 and $600 \mathrm{kPa}$, respectively. Test V6-600 clearly shows the most diffuse volumetric strains in the first increment 
and the most complex pattern of shear strain localisation with respect to the other two tests.

It follows that, differently from the test V1-0 performed without confinement, which exhibited axial splitting as main failure mode, the localization process of the specimens V3-50 and V6-600 are quite similar to those observed on either small specimens with horizontal fissures (and no confinement) (e.g. [21, 22]) or on unfissured specimens (e.g. [23]).

Similar results have been found, during confined (i.e., $\sigma_{3}=$ 50,300 and $600 \mathrm{kPa}$ ) plane strain compression tests, for specimens having horizontal fissures and fissures inclined at about $45^{\circ}$ to the horizontal.

\section{Conclusion}

This paper reports some of the results of plane strain tests carried out on specimens of an I6 scaly clay from Santa Croce di Magliano (South of Italy). The tests were conducted on REV specimens having vertical, medium and horizontal fissuring inclination, using different confining pressures $(50 \mathrm{kPa}, 300 \mathrm{kPa}, 600 \mathrm{kPa})$. Test results were also compared with previous tests carried out on the same material without confinement [10].

The standard stress-strain response analysis was accompanied by measurement of displacement and strain fields through 2D-DIC. The derived incremental strain fields have allowed the deformation processes to be followed throughout each test, providing both spatial and temporal strain evolution.

The results show that, irrespective of the inclination of fissures, the presence of confinement pressure during the tests increases the material strength and stiffness and changes the patterns of strain localisation with respect to those observed without confinement.

The pattern of behaviour emerging from the DIC results here presented is quite clear: both the inclination of fissures in the specimen and the confining pressure are playing a role in the evolution of the deformation within the material and, therefore, affect the global specimen response. DIC provided a detailed picture of the processes occurring throughout the tests, from the early stages, when both closure and opening of pre-existing fissures could be tracked, to the complete development of a sliding mechanism between near - rigid bodies. In particular, for vertical fissures, the presence of confinement makes it change the localisation processes, which become similar to those observed for unfissured clays: initial diffuse volumetric strains on the specimen photographed surface, first localised areas about the maximum shear stress along the stress-strain curve, further development of strain localisation in narrow areas and onset of sliding mechanism. On the contrary, for the tests without confinement, the pre-existing fissuring network was found to dictate the evolution of the deformation within the material that, therefore, exhibited the activation of multiple pre-existing sub-vertical fissures since the start of loading and the final failure mode was essentially axial splitting.

\section{References}

1. AGI, Some Italian experiences on the mechanical characterisation of structurally complex formations. International Society of Rock Mechanics (ed) Proceedings of the $4^{\text {th }}$ international congress of the international society of rock mechanics, Montreux, vol 1, 827-846 (1979)

2. A. Croce, Opening address of the Int. Symp. "The Geotechnics of structurally complex formations". Capri, II, 148-151 (1971)

3. K. Terzaghi, Stability of slopes in natural clays. In: Proceedings of the 1st conference on soil mechanics, Harvard, 1, 161-185 (1936)

4. F. Esu, Behaviour of slopes in structurally complex formations. The Geotechnics of Structurally Complex Formations, Proc. intern. symp., Capri, 2, 292-304 (1977)

5. F. Cotecchia, C. Vitone, F. Cafaro, F. Santaloia, The mechanical behaviour of intensely fissured high plasticity clays from Daunia. Second international workshop on characterisation and engineering properties of natural soils, 1975-2003, Singapore (2006)

6. A.G.I. Geotechnical properties and slope stability in structurally complex clay soils. Geotech. Engineering in Italy, 2, 189-225 (1985)

7. F. Cotecchia, C. Vitone, F. Santaloia, G. Pedone, O. Bottiglieri, Landslides J., 12 ,5, ISSN 1612-510X: 877-893 (2015)

8. C. Vitone, F. Cotecchia, Géotechnique, 61, 12, $1003-$ 1018 (2011)

9. C. Vitone, F. Cotecchia, G. Viggiani, S.A. Hall, Int. J. Num. An. Meth. Geomech., 37, 11, 1510-1534 (2013)

10. C. Vitone, F. Cotecchia, J. Desrues, G. Viggiani, Soils Found., 49, 355-368 (2009)

11. C. Vitone, G. Viggiani, F. Cotecchia, S.A. Hall, Acta Geotechnica, 8 ,3, 247-263 (2013)

12. F. Silvestri, C. Vitone, A. d'Onofrio, F. Cotecchia, R. Puglia, F. Santucci de Magistris, Geotechnical Soil Stress-Strain Behaviour: Measurement, Modelling, Analysis, Springer, the Netherlands, 333-350 (2007)

13. V. Nardelli, M.R. Coop, C. Vitone, Géotechnique letters, 6, 205-210, ISSN: 2045-2543 (2016)

14. L. Dazzaro, L. Rapisardi, Schema geologico del margine appenninico tra il F. Fortore e il F. Ofanto. Memorie della Società Geologica Italiana, 51, 143147 (1996)

15. A. Melidoro, G. Melidoro, V. Panaro, Deformazioni gravitative dei versanti nei terreni flisciodi con argille a bentoniti di Santa Croce di Magliano (Molise). Quarry \& Construction (2002)

16. C. Mondello, Global behaviour and localisation phenomena in complex fine grained soils, Master Thesis, Technical University of Bari (2018)

17. A. Castellano, Confining pressure and fissuring orientation: DIC analysis on natural clay., Master Thesis, Technical University of Bari (2017) 
18. S.D. Athanasopoulos, S.A. Hall, J.F. Kelleher, T. Pirling, J. Engqvist, J. Hektor, Mapping Grain Strains in Sand Under Load Using Neutron Diffraction, Micro to Macro, conference, University Mediterranea of Reggio Calabria, Italy (2018)

19. E. Tudisco, E. Andò, R. Cailletaud, A.S. Hall, TomoWarp2: A local digital volume correlation code. SoftwareX 6, 267-270 (2017)

20. E. Tudisco, S.A. Hall, E.M. Charalampidou, N. Kardjilov, A. Hilger, H. Sone, Full-field measurements of strain localisation in sandstone by neutron tomography and $3 D$-volumetric digital image correlation. 10 World Conference on Neutron Radiography (2014)

21. C. Vitone, F Cotecchia, G. Viggiani, Localisation processes and size effects for fissured clay specimens. Advanced Multiphysical Testing of Soils and Shales (AMTSS). In Springer Series in Geomechanics and Geoengineering, Laloui, Lyesse; Ferrari, Alessio (Eds.): 219-225 (2013)

22. C. Vitone, F. Cotecchia, A. Federico, G. Viggiani, It. Geotech. J., 2, 7-29 (2018)

23. G. Viggiani, J. Desrues, Experimental observation of shear banding in stiff clay. Geotechnical Innovations (studies in honour of Prof. Pieter A. Vermeer on occasion of his 60th birthday), R.B.J. Brinkgreve et al. Eds., Verlag Glückauf Essen, 649-658 (2004) 